\title{
Reverse Design and Rapid Prototyping ABS Part Assembly with Hard Material
}

\author{
Yaodong $\mathrm{XU}^{1, \mathrm{a}}$ \\ ${ }^{1}$ Department of Manufacturing Engineering, Shanghai University of Engineering \\ Science,Shanghai,200437, China \\ ${ }^{\text {a }}$ sodarbiscuit@163.com ${ }^{1}$
}

\begin{abstract}
In order to realize the variant design of the existing product, and rapid completion of the manufacturing and assembly, a new product was designed by reverse design method,and manufactured by rapid prototyping technology to finish assembly with hard material part. An experiment of rapid prototyping part with different model scales assembling with metal part was done to find the right scale of 1.008 of the model in $\mathrm{H} / \mathrm{h}$ tolerance fit and $0.1 \mathrm{~mm}$ more in size in interference fit.Through the static theory analysis, the amount of the interference fit was calculated by equal torque in contrast with mechanical assembly.The result was further proved by ProE mechanica simulation for stress and strain.Applying the rule of the results in experiment,prototyping part assembly with hard material part in different types of fit can be realized.
\end{abstract}

\section{Introduction}

With the manufacturing industry from mass production to customized production, product design needs shorten the design cycle,adapt to changing market, and be recognized by the market as soon as possible. The reverse design, also known as reverse engineering, is a process that can change 3-dimensional cloud point data obtained by $3 \mathrm{D}$ scanning measurement on existing physical model to the STL model, and further parametric transformation into CAD model through reverse design software [1]. Through reverse design, the original design intent of the physical model can be obtained which can be redesigned to complete a new product,that can greatly reduce the time and cost of forward design, and bring products to market in the most efficient way [2].

Rapid prototyping can directly convert the design into a physical part, which can be used as a model for customer evaluation of the design, and can be made for functional parts with a certain strength and toughness for the actual assembly. With the rapid prototyping technology and reverse engineering combination,the development cycle of new products from design to manufacturing can be greatly accelerated, that gets more and more extensive applications[3]. Due to rapid prototyping material itself shrinkage, it will produce a certain proportion of contraction when printed from the original CAD model.In some record, the

* Corresponding author: sodarbiscuit@163.com 
contraction of prototyping part, material shrinkage rate and filling rate in volume are related, and the relevant calculating formula of the contraction through the experimental data was obtained and reasonable scales about the model in prototyping in different assembly relationship with similar material was put forward . However, when the rapid prototyping part was used as a functional part,it will necessarily involve with assembly of different materials, such as ABS material and metal material assembly. This makes the assembly of rapid prototyping parts with hard material parts become a research topic in considering the material shrinkage and the printed model scale to meet different assembly relationship.

In this paper, through the experimental analysis, theoretical derivation and simulation verification, the result of rapid prototyping model scale and the assembly relationship was found and can be used as a reference for prototyping part assembly with hard material part,that is the innovation of this paper.

\section{Part Reverse Design}

Reverse Design.It can make reverse design by Geomagic Studio software on the STL model, that can be converted into CAD model ,saved as. iges files or .step files[4].STL is a overall model composed of many triangles that cannot be edited in CAD because of lack of feature parameters of surface.By Geomagic Studio surface parameterization,the STL model can be processed by extracting contour, region division according to surface automatic classification and surface fitting,as shown in Figure 1, and then the fitting surfaces can be seamed and converted into CAD model, which can be used in the later stage in CAD software design.

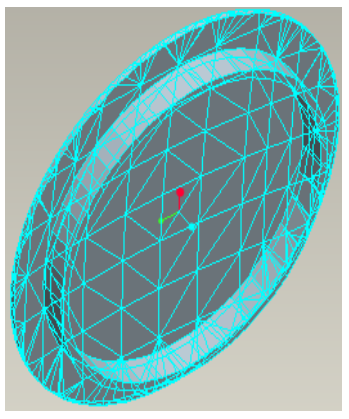

(a) Original STL model

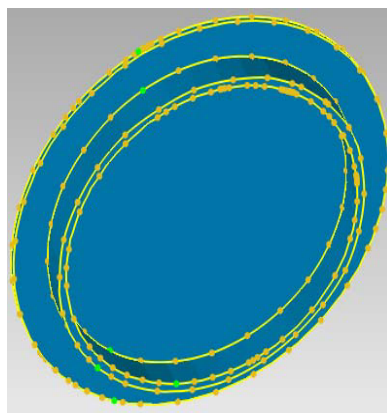

(b)Extracting contour and region division

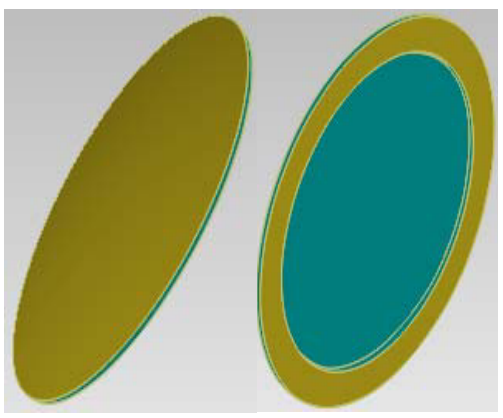

(c)Surface fitting

Fig.1. Geomagic Studio reverse design

Forward Design.In reverse design, the STL model should be put into parameterization as much as possible, but sometimes the parameter processing is not sufficient, or for the sake of simplicity, just several surfaces parameter processing. As shown in Figure1 (c), only a selection of three surfaces about the spherical, planar and cylindrical surface for parametric processing, other part of the model will be created by modeling through forward design.As shown in Figure2 (a), by ProE software forward design, the reverse design process was greatly simplified. In addition,through the forward design, it can retrofit and customize design, as shown in Figure2 (b), with a logo added on the surface of the sphere. 


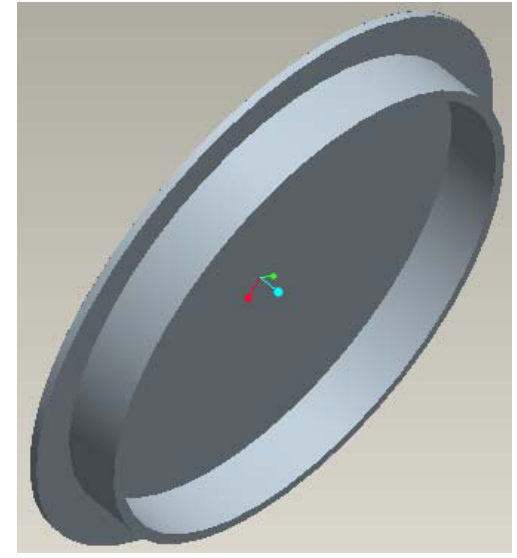

(a) Solid modeling

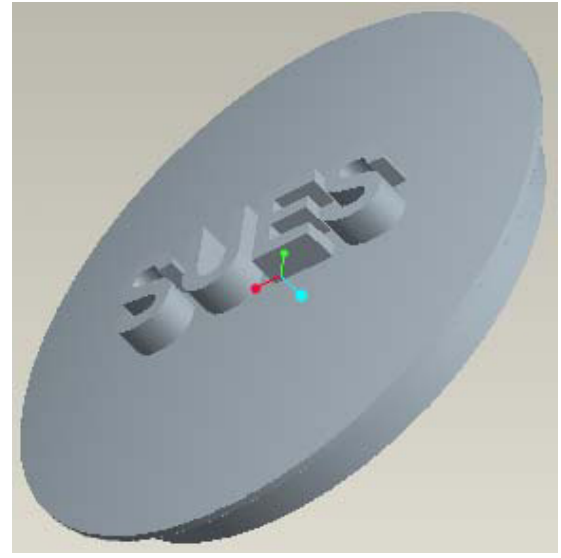

(b) Retrofit design

Fig.2. Forward design modeling

\section{Rapid Prototyping and Contraction Test}

After model reverse design, through rapid prototyping, part was made for the actual assembly. In order to determine suitable scale of the model to prototype,an experiment of ABS rapid prototyping parts assembly with the metal parts was carried out in different model scale. The model scale and assembly results, as shown in Table 1.

As shown in Table 1, scale of 1.008 of the model is the right value to achieve $\mathrm{H} / \mathrm{h}$ tolerance fit , that is no clearance fit,when the outer cylindrical surface of the prototyping part assembly with the inner cylindrical surface of the metal part.As analyzed,because the rapid prototyping part is a shell part, the material filling rate in volume is approximately $100 \%$ to be considered as a solid part, and the contraction of the part is mainly related to shrinkage rate of ABS material.As known in the experiment, ABS material shrinkage rate was $0.8 \%$, so printed by scale of 1.008 of the model, the size of the prototyping part after contraction is equal to the original model size. As the assembly part is hard metal,which has few deformation after machining,and the size of it coincides with the size of the ABS rapid prototyping part after contraction.

TABLE 1. MODEL SCALE AND ASSEMBLY RESULTS

\begin{tabular}{|c|c|c|c|}
\hline Model scale & 1 & 1.008 & 1.02 \\
\hline Model size/mm & 40 & 40.32 & 40.8 \\
\hline Assembly results & Much clearance & No clearance fit & Failure assembly \\
\hline
\end{tabular}

\section{Model Scale and Assembly with Hard Material}

Similar Assembly Relationship. When assembly relationship is similar, the model scale is determined by the dimension deviation of the assembly.For instance, the hole of the metal part in assembly is in taper,and the dimension range on both sides of the hole diameter is $0.2 \mathrm{~mm}$. Due to the basic cylinder diameter of the rapid prototyping part is $40 \mathrm{~mm}$,according 
to $40 \times 0.005=0.2 \mathrm{~mm}$, if one side of the hole in diameter can realize $\mathrm{H} / \mathrm{h}$ tolerance fit by the scale of 1.008 ,the other side must reduce scale of 0.005 from 1.008 in diameter, that is 1.003 ,to achieve the same type of $\mathrm{H} / \mathrm{h}$ tolerance fit,data of those was proved through experiment.

Different Assembly Relationship. When the assembly relationship is not the same,such as interference fit other than $\mathrm{H} / \mathrm{h}$ tolerance fit, it needs increase model scale. As recorded, plastic model of hole and shaft assembly interference margin is generally $0.2 \mathrm{~mm}$, based on basic dimension of $\phi 10 \mathrm{~mm}$ in assembly. As the plastic part assembly has bilateral deformation in hole and shaft,that when the plastic shaft contracts at diameter , the plastic hole expands at the same time. However, the plastic part and metal part assembly has single side deformation,that the plastic shaft contracts and the metal hole remains the same at diameter size, so the plastic part and metal part assembly interference margin should be $0.1 \mathrm{~mm}[5]$, as refered $1 / 2$ of $0.2 \mathrm{~mm}$ as before.According to $40 \times 0.0025=0.1 \mathrm{~mm}$, the interference fit was achieved by 1.0105 scale when the $\mathrm{H} / \mathrm{h}$ tolerance fit by 1.008 scale, however the clearance fit was achieved by 1.0055 scale to realize free rotation at the fit level.The model scale and assembly relationship ,as shown in Table 2.

TABLE 2. MODEL SCALE AND ASSEMBLY RELATIONSHIP

\begin{tabular}{|c|c|c|c|}
\hline Model scale & 1.0055 & 1.008 & 1.0105 \\
\hline Model size/mm & 40.22 & 40.32 & 40.42 \\
\hline Assembly relationship & Clearance fit & $\mathrm{H} / \mathrm{h}$ tolerance fit & Interference fit \\
\hline
\end{tabular}

\section{Stress and Strain Analysis}

Static Theoretical Derivation.According to the formula of elastic modulus, as shown in formula 1 ,stress $\sigma=\frac{F}{S}$, strain $\varepsilon=\frac{\Delta d}{d}$

$$
E=\frac{\sigma}{\varepsilon}
$$

Diameter of cylindrical surface of steel $d=40 \mathrm{~mm}$,Elastic modulus of steel $E=200 \mathrm{GPa}=2 \times 10^{5} \mathrm{MPa}$, Interference margin $\Delta d=2 \mu \mathrm{m}[6]$.

The uniform load of the cylinder is obtained as formula 2.Data input,conclude $\sigma=10 \mathrm{MPa}$.

$$
\sigma=E \cdot \varepsilon=E \cdot \frac{\Delta d}{d}
$$

The differential element force on the cylindrical surface is $\mathrm{d} F$, the friction coefficient of the contact surface is $f$, and the contact area of the force element is $\mathrm{d} S . l$ is the width of the cylindrical surface, $r$ is the radius of the cylindrical surface, and schematic diagram of the differential element force is shown in Figure 3.

$$
\mathrm{d} S=\operatorname{lr} \mathrm{d} \theta
$$

The torque generated by the interference fit can be calculated by formula 4.It is found that the torque is related to the uniform load and friction coefficient of the contact surface.

$$
T=\int r \cdot f \mathrm{~d} F=\int r \cdot f \sigma \mathrm{d} S=\int_{0}^{2 \pi} r \cdot f \sigma \cdot l r \mathrm{~d} \theta=2 \pi f \sigma l r^{2}
$$


It is known ABS elastic modulus $E=2 \mathrm{GPa}=2 \times 10^{3} \mathrm{MPa}$ (value higher than the same of ABS resin due to added PC ingredients in material) ,1/100 of the elastic modulus of steel,which is $200 \mathrm{GPa}$. Take into account the same effect of equal torque in interfere fit, that the torque keeps the same, namely $f \sigma$ keeps the same. Considering the friction coefficient of

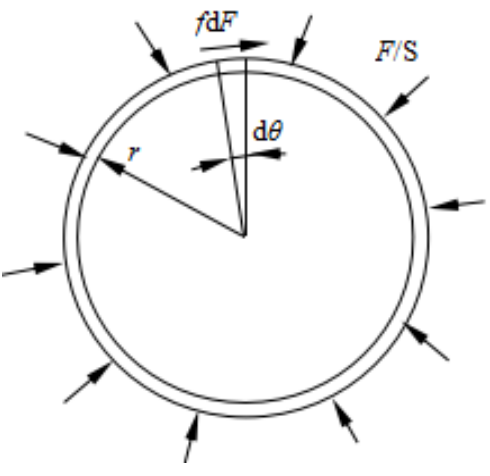

Fig.3. Differential element diagram

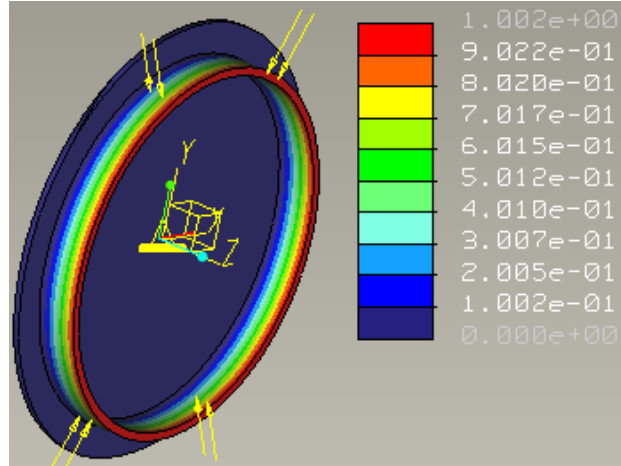

Fig.4. Displacement in static modal steel and steel is 0.2 , the friction coefficient of ABS and steel is 0.4 , ABS material radial stress in interfere fit is half that of steel, namely $5 \mathrm{MPa}$. The data are substituted into the formula 2, conclude $\Delta d=0.1 \mathrm{~mm}$.

Because the steel parts assembly has bilateral deformation in hole and shaft fit, if the actual interference fit margin is $2 \mu \mathrm{m}$, the interference deviation is $4 \mu \mathrm{m}$ in consulting reference handbook.But when ABS material and steel assemble in shaft and hole, ABS will produce contraction and hard material of steel will not contract, so ABS rapid prototyping part assembles with hard material in interference fit, under the condition of the same torque, the cylindrical surface of the ABS rapid prototype part will produce contraction of $0.1 \mathrm{~mm}$ in diameter,calculated by formula 2 . This result coincides with the experimental data shown in Table 2,calculated by $40.42-40.32=0.1 \mathrm{~mm}$.

Simulation Analysis.Applying Proe mechanica software to make stress and strain modal analysis of the rapid prototype model[7], assigning ABS for model material,elastic modulus $E=2000 \mathrm{MPa}$, poisson ratio $\mu=0.394$, uniformly distributed load of $5 \mathrm{MPa}$, constraint at the bottom of the cylinder,stress and strain simulation under static load was done[8], as shown in Figure 4,display of deformation displacement. At the bottom of the cylindrical surface, the dark blue area shows the displacement deviation is $1.002 \mathrm{e}-01 \mathrm{~mm}$, that is approximately $0.1 \mathrm{~mm}$, which is in exactly consistent with the theoretical calculation results.Due to the cylindrical surface deformation like as cantilever beam structure, from the bottom of the constraint farther along the axis of cylindrical surface, the deformation is bigger.

\section{Summary}

Using the reverse and forwards design method, to reverse and retrofit design, it can achieve a new product design. When rapid prototyping part is taken into the structure of the assembly, the prototyping model scale is firstly determined to meet the $\mathrm{H} / \mathrm{h}$ tolerance fit according to the material shrinkage rate and filling rate in volume, then the suitable scale is selected according to the similar assembly relationship or different one. As to similar assembly relationship, it only needs change the value of rigid dimension according to the deviation of assembly.And as to different relationships of assembly, when varying from the $\mathrm{H} / \mathrm{h}$ tolerance fit to interference fit for rapid prototyping of plastic part assemble with hard material, the interference fit needs surplus of $0.1 \mathrm{~mm}$ in rapid prototyping model size based on $\mathrm{H} / \mathrm{h}$ tolerance fit,otherwise, a clearance fit needs reduce the allowance of $0.1 \mathrm{~mm}$ based on 
$\mathrm{H} / \mathrm{h}$ tolerance fit.

\section{Acknowledgement}

In this paper, the research was sponsored by Shanghai Municipal Education Commission Key Project(Project No. BJYXX13YYYJ00) and Shanghai University Experimental Technology Team Construction Plan(Project No. A2-8950-13-0706).

\section{References}

1. Huilan Wu,Peng Song.Experiment of the Curved Surface Reverse Reconstruction Based on Non-contact Laser Scanning[J].Research and Exploration in Laboratory, 2014,33(7):44-47.

2. Lijing Liu,Hui Yang.3D reverse engineering design on seed tube based on Geomagic Design software[J].Transactions of the Chinese Society of Agricultural Engineering,2015,31(11):40-43.

3. Jiechun Yan,Dingyin Feng.Redesign of product based on reverse engineering and rapid prototyping technology[J].Tool Engineering,2015,49(7):85-87.

4. Min Cai,Siyuan Cheng,Xue Yang,etc.Study of Feature Modeling Based on Geomagic Studio[J].Machine Tool \& Hydraulics,2014,42(21):142-145.

5. Jiqiang Tang,Yongbin Zhang,Libin Zhao.Interference fitted metal-composite material flywheel rotor[J].Optics and Precision Engineering,2013,21(11):2639-2647.

6. Hongbin Yi.Mechanical product inspection and quality control[M].Beijing:Chemical Industry Press, 2011.

7. Zegang Sun.Fatigue Analysis on Key Part of Socket Connector Binding Tool Based on Proe/Mechanica[J].Coal Mine Machinery,2012,33(9):128-130.

8. Zengqiang Tian,Decong Zheng,Yuming Guo.Statistic and modal analysis of the gear-housing[J].Journal of Chinese Agricultural Mechanization,2013,34(4):178-181. 